\title{
Aesistencia al cizallamiento de un sistema rolalmente cerámico frente a siete sistemas ceramo-metálicos: estudio comparativo
}

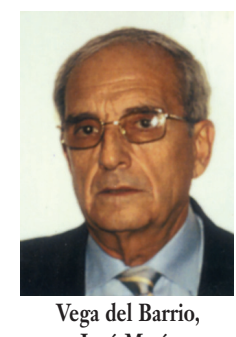

José María

\author{
Shear resistence of one all-ceramic sustem versus seven ceramo-metallic sustems: \\ a comparative studu
}

Vega del Barrio, José María

Profesor Titular. Materiales Odontológicos. Universidad Complutense de Madrid. Facultad de Odontología. Departamento de Estomatología II.

El presente trabajo se ha realizado mediante contrato de investigación al amparo del artículo 83 de la ley de Universidades y de los Estatutos de la U.C.M.

\section{Correspondencia}

José Ma Vega del Barrio

Facultad de Odontología

Universidad Complutense de Madrid

Pl. Ramón y Cajal s/n

28040 - Madrid
Resumen: En el presente trabajo se mide y compara la resistencia al cizallamiento de la unión ceramocerámica, de un nuevo sistema totalmente cerámico, de óxido de zirconio y su cerámica específica, frente a siete sistemas ceramo-metálicos de los cuales dos son uniones de cromo-niquel a cerámica, cuatro de titanio colado a cerámica y uno de cobalto-cromo a cerámica.

Se utilizaron para cada grupo 10 cilindros de dimensiones adecuadas para expresar los resultados en megapascales. Una de las bases de cada cilindro fue recubierta por una capa de la cerámica respectiva de $1 \mathrm{~mm}$ de espesor $\mathrm{y}$, después, procesada en el horno. Posteriormente se sometieron a una prueba de cizallamiento para medir la resistencia de la unión. Se investigó un total de 80 muestras.

En los resultados los valores medios mas altos de la resistencia de la unión, expresados en MPa, se han obtenido con el sistema totalmente cerámico, identificado como grupo VI $(47,82 \mathrm{MPa})$, seguidos de los de cobalto-cromo a cerámica (grupo VII: $41,82 \mathrm{MPa}$ ) y de los de niquel-cromo a cerámica (grupo SN: 40,16 MPa y grupo V: 40,32 MPa). Los valores mas bajos se obtuvieron con los sistemas titanio colado a cerámica (grupo III: 37,49 MP; grupo IV: 30,74MPa; grupo II: 29,16 MPa y grupo I: 26,18 $\mathrm{MPa})$. Se realizaron los tests de ANOVA y Student Newman-Keuls $(p<0,05)$, encontrándose diferencias estadísticamente significativas entre el grupo VI y los grupos I, II y IV.

Palabras clave: Resistencia de la unión totalmente cerámica, Resistencia de la unión ceramometálica, Cercon, Óxido de zirconio.

Abstract: The present paper measures and compares the shear resistance of the ceramo-ceramic junction of a new all-ceramic system (made of zyrconium oxide and its specific ceramic) as opposed to seven ceramometalic systems, out of which two are unions made of two combinations between nickel-chromium alloys and ceramics, four consist of cast titanium plus ceramics and one comprises cobalt-chromium and ceramics.

For each group, 10 cylinders of appropriate dimensions were used in order to show the results in Mpa. One of the bases of each cylinder was covered by a layer of the relevant ceramic of $1 \mathrm{~mm}$ thickness and subsequently processed in the oven. Afterwards they underwent a shear resistance test to measure the junction resistance. A total number of 80 samples were tested. The group VI samples correspond to the testing material (all-ceramic system).

Results: the highest average values of the shear resistance (showed in Mpa) were given by the all-ceramic system -identified as group VI (47.82 Mpa)-, followed by the cobalt-chromium alloy to ceramic (group VII: $41.82 \mathrm{Mpa}$ ) and the nickel-chromium alloys to ceramic (SN group: $40.16 \mathrm{Mpa}$ and group V: $40.32 \mathrm{MPa}$ ). The lowest values were given by the cast titanium to ceramic systems (group III: 37.49 Mpa; group IV: $30.74 \mathrm{Mpa}$ group II: $29.16 \mathrm{Mpa}$, and group I: $26.18 \mathrm{Mpa}$ ). ANOVA and Student Newman-Keuls calculations $(p<0.05)$ were applied. Statistically significant differences were found between group VI and groups I,II and IV.

Key words: Shear resistance of all-ceramic system, Shear resistance of ceramometalic systems, Cercon, Zirconium oxide.

BIBLID [1138-123X (2005)10:5-6; septiembre-diciembre 497-640]

Vega del Barrio JM. Resistencia al cizallamiento de un sistema totalmente cerámico frenta a siete sistemas ceramo-metálicos: estudio comparativo. RCOE 2005;10(5-6):529-539. 


\section{Introducción}

En los últimos años hemos asistido a una verdadera revolución en el mundo de los materiales cerámicos. Independientemente de que existen muchas aportaciones, nosotros vamos a centrar la atención aquí en los sistemas que han sustituido el metal por cerámica en las técnicas ceramometálicas. Se ha cambiado la convencional cofia o estructura metálica por estructuras "base» totalmente cerámicas sobre las que se cuece la cerámica convencional. ¿Es suficientemente resistente la unión entre las dos cerámicas? Las fuerzas tangenciales, de deslizamiento o de cizallamiento, aparecen como pruebas muy idóneas para estudiar la resistencia de las uniones entre materiales. Hasta ahora no han sido muy utilizadas, pero últimamente se llama la atención, desde diferentes sectores, sobre su evidente interés, ya que este tipo de solicitudes mecánicas está muy presente en la fisiología de todo el complejo estomatognático. A pesar de que muchos metales conservan excelentes propiedades mecánicas, actualmente se asiste a un lento decrecer en el interés por los mismos en todo el campo dental. Los condicionantes que presiden hoy las indicaciones de los metales y las cerámicas en OdontologíaEstomatología son múltiples. Por un lado las incógnitas y reservas con respecto a las heterogeneidades metálicas en el medio bucal; por otro lado el precio de las denominadas "preciosas»; por otro la enorme presión hacia la denominada "odontología estética» y, por último, la irrupción del titanio, lo que ha originado una cantidad, progresivamente creciente, de pa- cientes que ya son portadores de implantes de titanio 0 de alguna de sus aleaciones. El último argumento indicado -la presencia creciente de pacientes con implantes de titanio en su boca- es una idea sobre la que, en general, estimamos se ha meditado poco. El futuro camina hacia una situación en que la presencia del titanio en muchas bocas hará reconsiderar la indicación de otros metales y aleaciones. Por otra parte, las aleaciones de níquel-cromo, tan extendidas en uniones ceramo-metálicas, a pesar de su excelente rigidez para situaciones de importante compromiso mecánico, sufren continuas controversias a causa de los problemas de biocompatibilidad que puede presentar el níquel. Ello ha originado que se hayan intentado con éxito otras alternativas para unir cerámica y metal, como paladio-plata, cromo-cobalto, el propio titanio, etc. Por todo ello, la alternativa de restauraciones totalmente cerámicas es del máximo interés ${ }^{15^{*}, 6^{*}}$.

\section{Hipótesis y objeos del presente estudio}

Dado que por una parte existen diferentes técnicas y materiales para unir cerámicas a metales y que, por otra, han surgido las modernas cerámicas para unir a otras cerámicas, nos proponemos demostrar si, desde el punto de vista de la resistencia al cizallamiento, la unión ceramo-cerámica de un nuevo sistema (Cercon Smart Ceramic $(\mathbb{B})$ es lo suficientemente resistente para considerarla una alternativa frente a las convencionales ceramo-metálicas cuya base metálica está constituida por níquel-cromo, por titanio o por cromo-cobalto utilizadas algunas durante muchos años y de las que existe amplia experiencia. Para ello se formulan los siguientes objetiVOS:

10. Medir la resistencia al cizallamiento de la unión de estructuras ceramo-cerámicas realizadas con un sistema moderno a base de oxido de zirconio y su cerámica específica (Cercon Smart Ceramics ${ }^{\circledR}$ de la Compañía DeguDent).

$2^{\circ}$. Medir la resistencia al cizallamiento de dos sistemas cromo-níquel + cerámica.

30. Medir la resistencia al cizallamiento de cuatro sistemas titanio colado + cerámica

$4^{0}$. Medir la resistencia al cizallamiento de un sistema cromo-cobalto + cerámica

$5^{\circ}$. Comparar los resultados de la resistencia al cizallamiento de la unión ceramo-cerámica frente a las diferentes uniones ceramo-metálicas estudiadas.

\section{Materiales, instrumentos प metodología}

El trabajo se ha diseñado de forma que en un laboratorio se prepararon y elaboraron las ochenta muestras (10 de cada grupo) (Laboratorios Aragoneses S.L. de Madrid) mientras que en otro laboratorio se procedió a las pruebas de cizallamiento, así como a su valoración y análisis de los resultados (Laboratorio de Investigación del Departamento de Estomatología II de la Facultad de Odontología de la Universidad Complutense de Madrid). 
En este trabajo se ha procedido bajo un método doble ciego; esto es, el laboratorio preparador elaboró las muestras pero solo facilitó al laboratorio experimental una clave identificativa de las mismas, guardando el nombre de las aleaciones y porcelanas hasta el final del experimento. Externamente las probetas no eran identificables por el aspecto, salvo, obviamente, las totalmente cerámicas.

\section{Materiales para la prepa- ración de las muestras}

Se han preparado un total de 80 probetas de las cuales 70 eran ceramo-metálicas, en diferentes combinaciones, y 10 ceramo-cerámicas de un sistema totalmente cerámico (Cercon Smart Ceramics $\left.{ }^{\circledR}\right)$ cuyo material de base es óxido de zirconio presinterizado. Se han obtenido un total de 8 grupos diferentes de diez probetas cada uno. En la figura 1 aparecen fotografiadas 10 probetas ceramometálicas y en la figura 2 las 10 ceramo-cerámicas. Se les asignó un código identificador que, como se ha indicado, solo se desveló al final de los resultados. En la tabla 1, se indican, de forma resumida, las composiciones genéricas de los grupos estudiados y comparados.

\section{Probetas ceramo-metálicas}

De cada grupo se prepararon 10 cilindros macizos de $8 \mathrm{~mm}$ de diámetro por $15 \mathrm{~mm}$ de altura, por el procedimiento a la cera perdida. Posteriormente, se modeló y se coció en el horno, sobre una de las bases del cilindro, una faceta de porcelana del mismo diámetro y de aproximadamente un milímetro de espesor (ver esquema de la figura 3). Los materia-

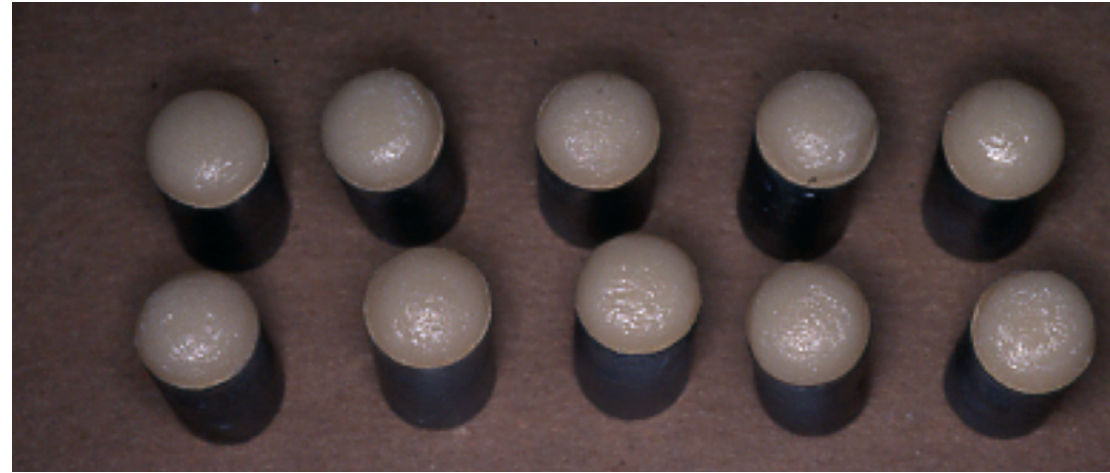

Figura 1. Fotografía de los 10 especímenes del grupo V, mostrados a título de ejemplo, utilizados en el presente ensayo.

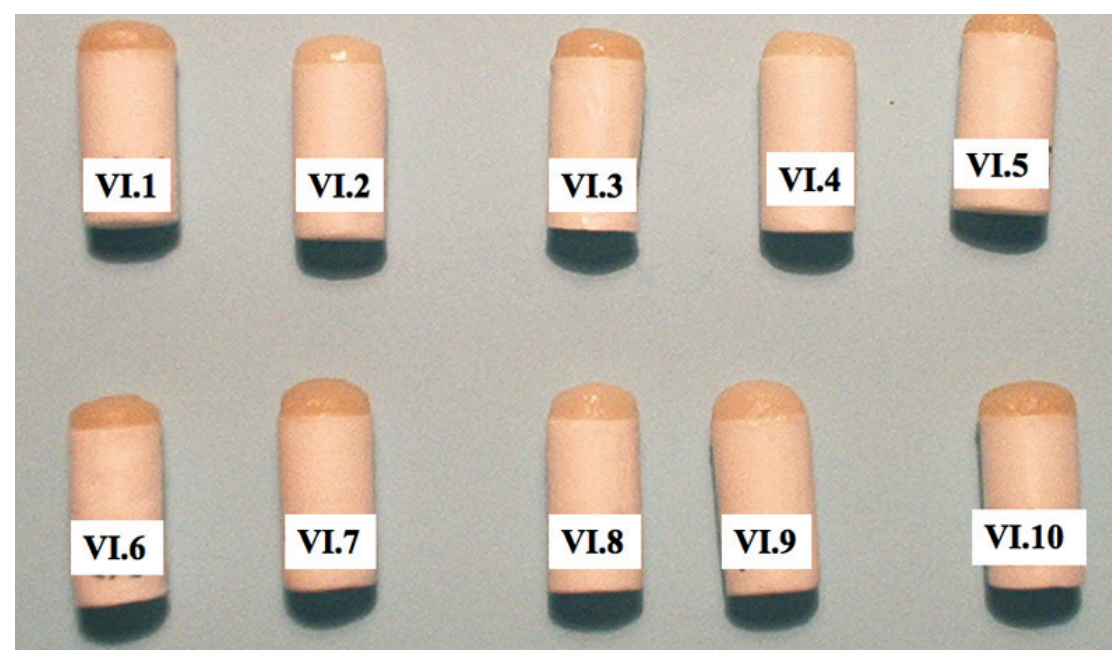

Figura 2. Fotografía de los 10 especímenes del grupo totalmente cerámico utilizados en el presente ensayo.

\begin{tabular}{|c|c|}
\hline id & 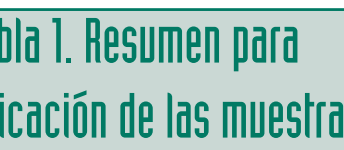 \\
\hline SN & Ní-Cr + cerámica. \\
\hline | & Titanio + cerámica \\
\hline$\|$ & Titanio + cerámica \\
\hline III & Titanio + cerámica \\
\hline IV & Titanio + cerámica \\
\hline V & Ní-Cr + cerámica. \\
\hline VI & Cercon base + Cercon \\
\hline & Ceram S ICercon Smart \\
\hline & Ceramics. \\
\hline VII & Co-Cr + cerámica. \\
\hline
\end{tabular}

les e instrumentos utilizados para su elaboración fueron los siguientes:

Dos sistemas níquel-cromo + cerámica

Probetas SN. El metal utilizado fue Ticonium (T-3 C \& B alloy). Es una aleación de concepción clásica que lleva muchos años en el mercado. La compañía preparadora declara como composición: níquel-cromo 90,5\%. La cerámica incorporada a una de las bases de estos diez cilindros fue Ceramco ॥ de Dentsplay. 


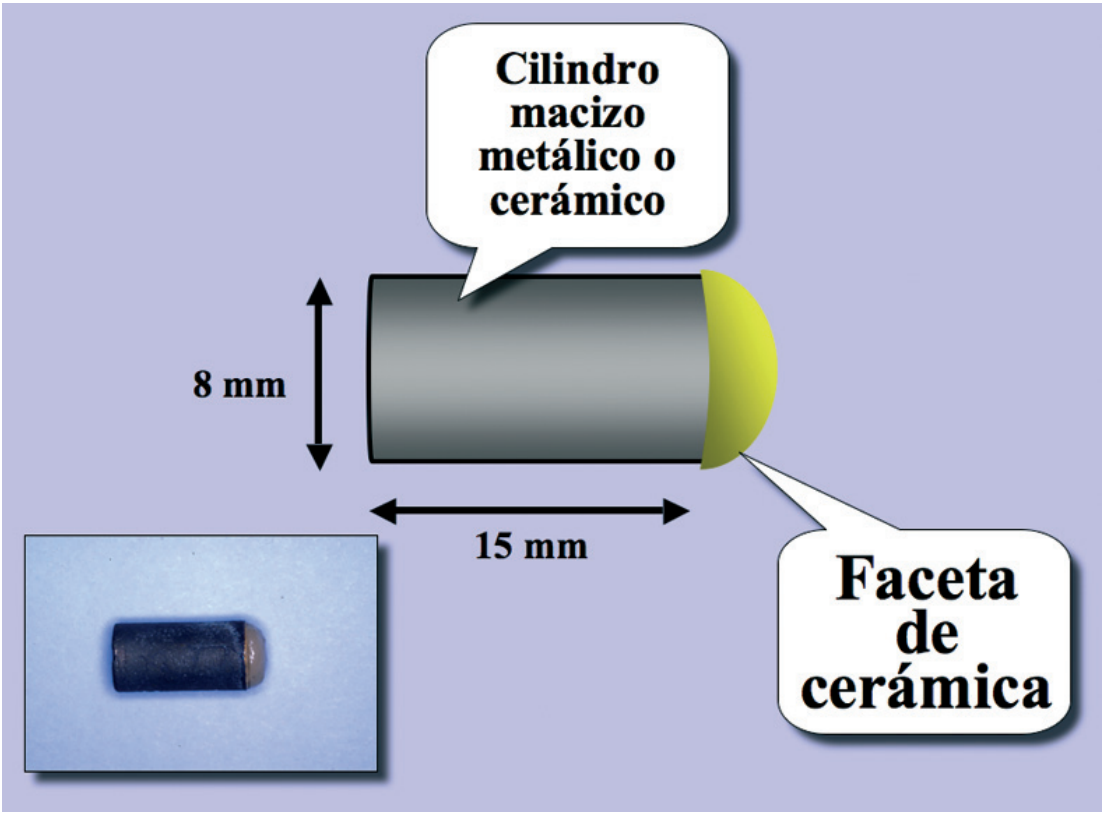

Figura 3. Esquema de la constitución de los cilindros experimentales. En el recuadro inferior izquierdo fotografia de uno de ellos.

Probetas V. El metal utilizado fue Tilite (Talladium Inc.). Se trata de una aleación, más moderna que la anterior que incorpora una pequeña cantidad de titanio. Composición declarada: $\mathrm{Ni}$ (60-76\%), Cr (12-21\%), Mo (4-14\%), Ti (4-6\%) La cerámica incorporada a una de las bases de estos cilindros fue Ceramco II de Dentsplay.

Material común en ambos grupos: La cera utilizada para elaborar ambos tipos de patrones fue la Nr. 40070 Bego best, habitual para coronas. El revestimiento utilizado, en ambos casos, fue CB-30 high heat crown \& bridges investment (Ticonium). Se trata de un revestimiento de oxifosfato (libre de carbono) de grano muy fino. Para el colado se utilizó el horno Eli Manfredi Aurum. Para la posterior oxidación del metal, previa a la colocación de la porcelana, se utilizó el horno Multimat- $C$ de Dentsplay. La cerámica empleada fue Ceramco II de
Dentsplay que fue procesada en horno Mach 3 Dentsplay.

Cuatro sistemas titanio mas cerámica. Se emplearon cuatro sistemas diferentes, identificados como probetas I, probetas II, probetas III y probetas IV:

Probetas I. Se trataba de un sistema experimental de prueba (no comercializado como tal). La parte metálica de las probetas era titanio puro Rematitán de la compañía Dentaurum. La cerámica fue Triceram ${ }^{\circledR}$ específica también de Dentaurum pero en vez de utilizar el bonding Dentaurum se utilizó un bonding de Heraeus-Kulzer. En otras palabras, se mezclaron voluntariamente componentes de marcas diferentes. La cera utilizada para elaborar los patrones fue la Nr. 40070. (Bego Best) habitual para coronas. El revestimiento empleado fue el Revestimiento Rematitán ${ }^{\circledR}$ Ultra. Para el colado se utilizó el aparato Cyclarc /l de J. Morita Co., que sucesivamente funde y cuela el metal. La porcelana se coció en el horno Multimat Mach 3 de Dentsplay. El bonding empleado en este caso fue Blendgold de HeraeusKulzer. Se insiste que, en este caso, deliberadamente, se utiliza algo que no es específico o propio del sistema. Se trata de un agente de unión, en forma de pasta, constituido por pequeñas partículas esferoidales de oro suspendidas en un vehículo adecuado de naturaleza orgánica.

Probetas II. Se trata de un sistema específico de titanio a cerámica propio de la compañía Vita. La cera empleada para elaborar los patrones fue la $\mathrm{Nr}$. 40070. Bego best, habitual para coronas. El revestimiento empleado para estos patrones fue el revestimiento Rematitán Ultra. El metal utilizado para obtener los colados fue titanio puro Vitatitan de Vita. Para el colado se utilizó la misma máquina que en el caso precedente. El material cerámico fue Vitakeramik de Vita, del que se aplicó una capa de $1 \mathrm{~mm}$ de espesor a una de las bases de cada cilindro metálico. Posteriormente horneada en el horno Multimat Mach 3 Dentsplay. El bonding fue el específico del sistema Vita.

Probetas III. Se trata del sistema específico de titanio a cerámica Rematitan -Triceram de Dentaurum junto con su bonding específico. La cera, el revestimiento y el procedimiento para la fusión y el colado fueron los mismos que en el caso precedente. El metal utilizado fue Rematitan (titanio puro Dentaurum). La cerámica fue Triceram (Dentaurum). Posteriormente horneada en el horno Multimat Mach 3 Dentsplay.

Probetas IV. Se trata nuevamente de una combinación experimental (es 


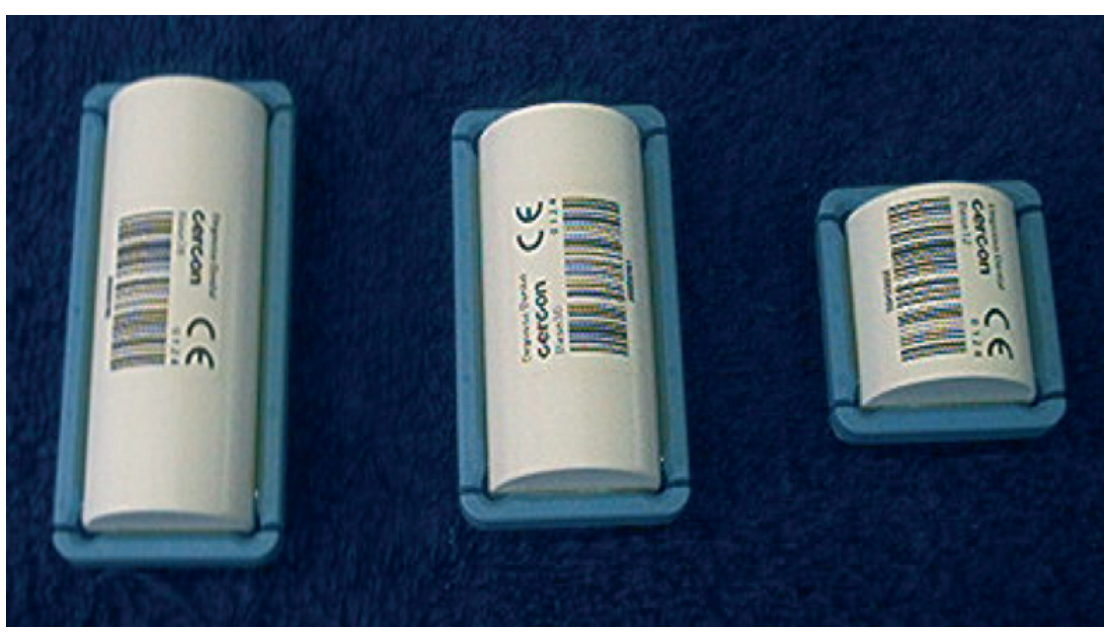

Figura 4. Fotografía de tres bloques presinterizados, de óxido de zirconio, tal como los presenta el fabricante, aptos para el mecanizado. En la presente investigación se utilizaron los mas pequeños.

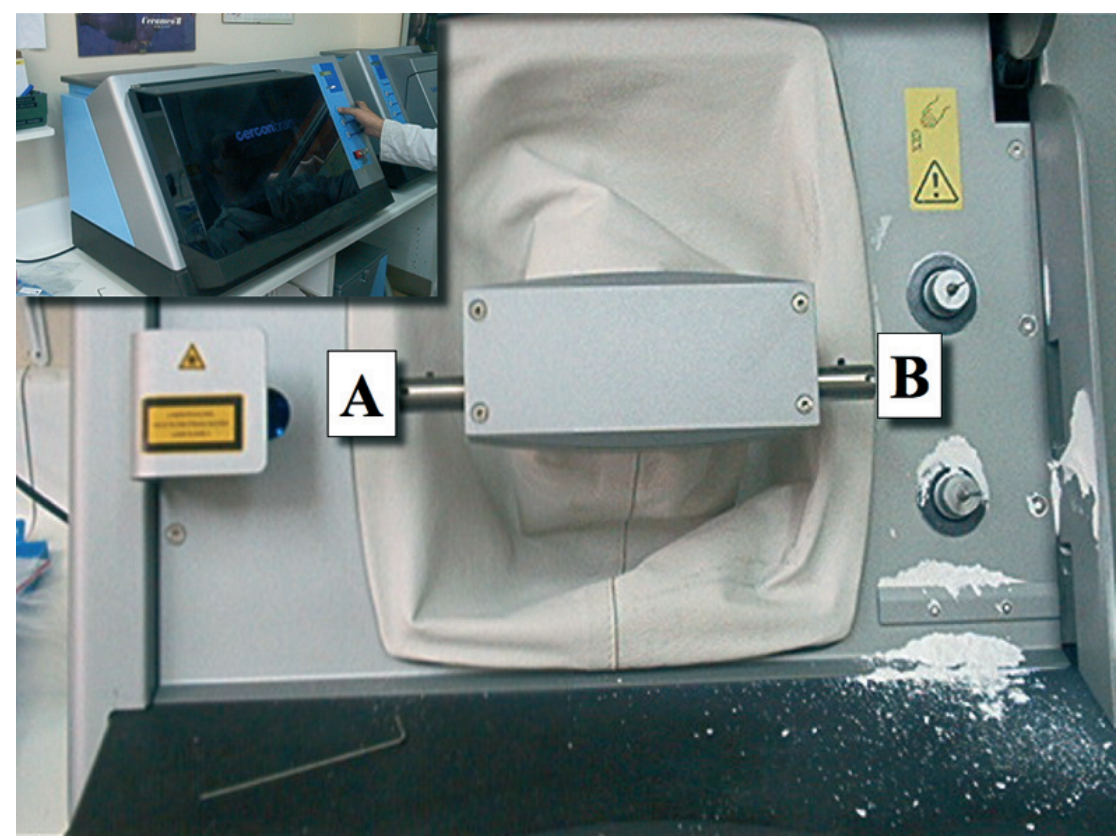

Figura 5. Fotografía de la máquina del sistema Cercon Smart Ceramics para mecanizar los bloques presinterizados de óxido de zirconio. En la parte señalada con "A" se realiza la lectura óptica del modelo o patrón. En la parte " $B$ " se realiza el procesado mecánico.

decir, no comercializada) de titanio con una cerámica y un bonding de prueba. La parte metálica de las probetas era titanio puro Rematitán de la compañía Dentaurum. La cerámica fue Noritake (Noritake Kizai). Como bonding se utilizó el de Heraeus-Kulzer, descrito anteriormente en el caso de las Probetas I. En otras palabras, se mezclaron voluntariamente componentes de fabricantes diferentes. La cera, el revestimiento y el procedimiento para la fusión y el colado fueron los mismos que en el caso precedente. Se aplicó una capa de cerámica de $1 \mathrm{~mm}$ de espesor sobre una de las bases de cada cilindro metálico. Posteriormente curadas en el horno Multimat Mach 3 Dentsplay. Se insiste que, en este caso, deliberadamente, se utilizaron también materiales pertenecientes a diferentes sistemas

Un sistema cobalto-cromo mas cerámica:

Probetas VII. El metal utilizado fue la aleación Sheradent de la firma Shera, cuya composición es Co-67\%, Cr-21\%, Mo- $6 \%$ y $\mathrm{W}-6 \%$. La porcelana fue igualmente Ceramco II de Dentsplay. Se procedió en idéntica manera que en el caso de las aleaciones níquel-cromo + cerámica.

\section{Probetas ceramo-cerámicas totalmente cerámicas}

Probetas VI. Se prepararon 10 cilindros macizos de Cercon Smart Ceramics mediante procesado mecánico, asistido por ordenador (procedimiento y maquinaria específicos de DeguDent) a partir de patrones de cera cuyas dimensiones fueron de $8 \mathrm{~mm}$ de diámetro por $15 \mathrm{~mm}$ de altura. Posteriormente se sinterizaron en el horno específico para este sistema de la compañía DeguDent. La cera utilizada fue la misma que en todos los casos precedentes.

La cerámica Cercon base se presenta en bloques presinterizados de óxido de zirconio suministrados por DeguDent (fig. 4). En el caso presente se utilizaron 
bloques del tamaño pequeño, mecanizados en la máquina especialmente diseñada para escaneado y fresado, específica del sistema Cercon Smart Ceramics. Dicha máquina consta de dos partes, representadas en la figura 5 con las letras A y B. En la parte A se coloca el patrón que hay que reproducir y todo el conjunto se desplaza automáticamente hacia la izquierda; un lector óptico «lee» el patrón. Posteriormente todo el conjunto se desplaza hacia la derecha (parte B) donde se ha colocado el cilindro de la cerámica presinterizada (fig. 6). Un sistema de mecanizado, constituido por dos fresas de diferente calibre, talla y reproduce la forma diseñada. Este bloque cerámico, una vez mecanizado, se lleva al horno para su sinterización. Es de destacar que el alto grado de contracción que sufre durante este proceso ya ha sido calculado y compensado previamente por la máquina durante la lectura óptica.

A continuación se modeló sobre una de las bases del cilindro cerámico (como en el caso de los cilindros metálicos) una faceta de la cerámica de recubrimiento Cercon Ceram $\mathrm{S}$ de $1 \mathrm{~mm}$ de espesor, una cerámica específica del sistema (Cercon Smart Ceramic), la cual fue nuevamente procesada en el horno específico del sistema.

\section{Metodología de la prueba de resistencia al cizalla- miento}

Toda la instrumentación descrita a continuación se encuentra en el laboratorio de investigación del Departamento de Estomatología II de la Facultad de Odontología de la Universidad Complutense de Madrid.

- Máquina universal para ensayos mecánicos, preparada para ejecutar

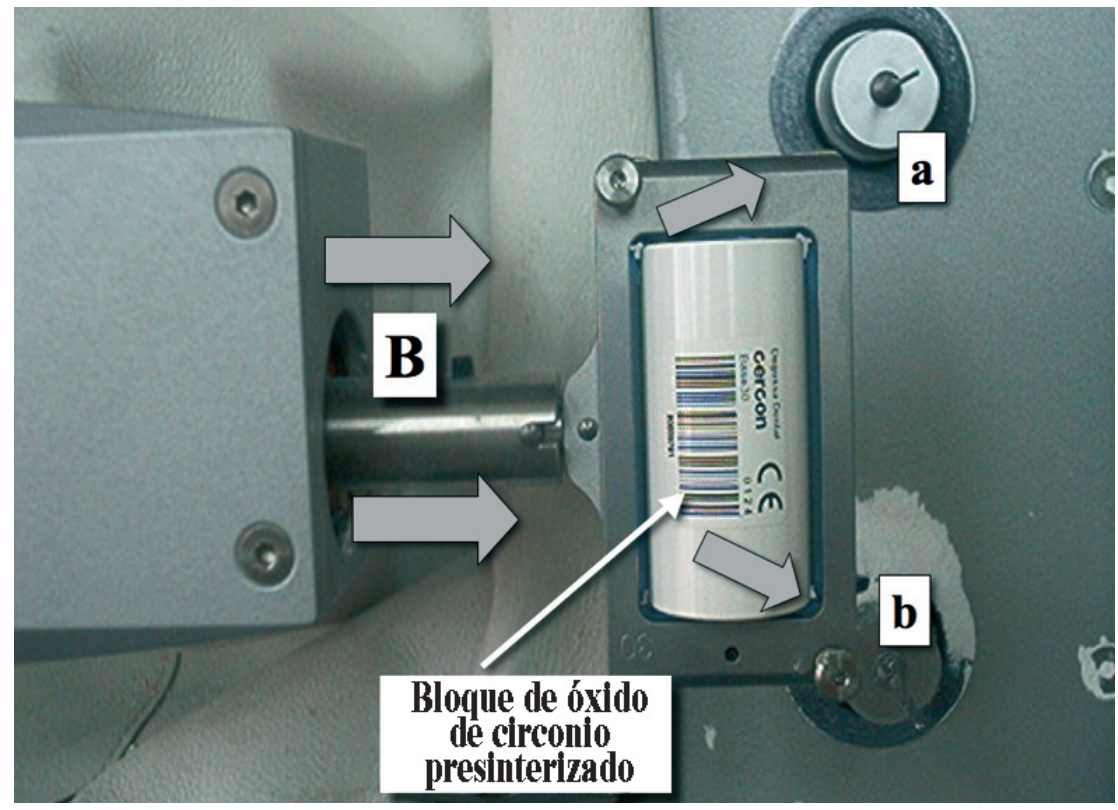

Figura 6. Detalle de la parte " $B$ " de la máquina representada en la figura anterior, con el bloque de óxido de zirconio dispuesto para ser mecanizado mediante las fresas representadas como " $a$ " $y$ " $b$ ".
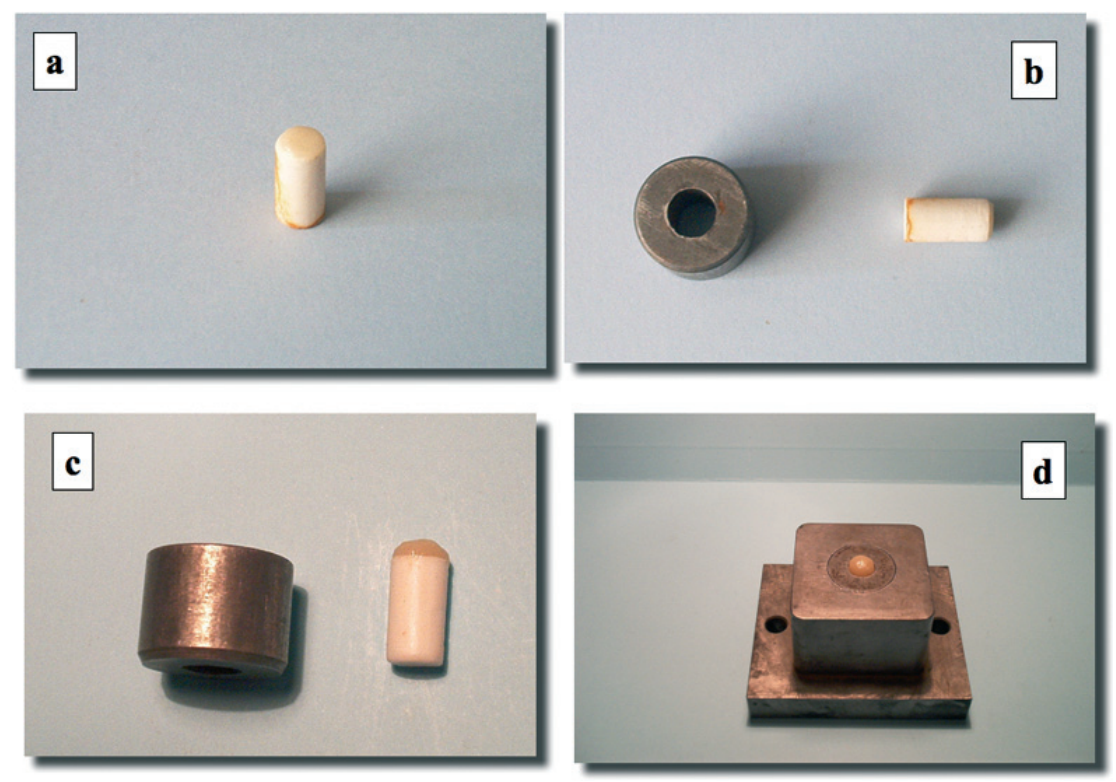

Figura 7. En "a" una muestra ceramo-cerámica entes del ensayo. En "b", "c" y " $d$ " dispositivos utilizados para alojar los especimenes destinados a la prueba de cizallamiento.

ensayo de cizallamiento (Hounsfieldtest H-Ti Equipment, Croydon). Alojamiento para las muestras, específica- mente diseñados para este tipo de pruebas, (figs. 7 y 8). La plataforma «d» de la figura 7, se acopla posteriormente 


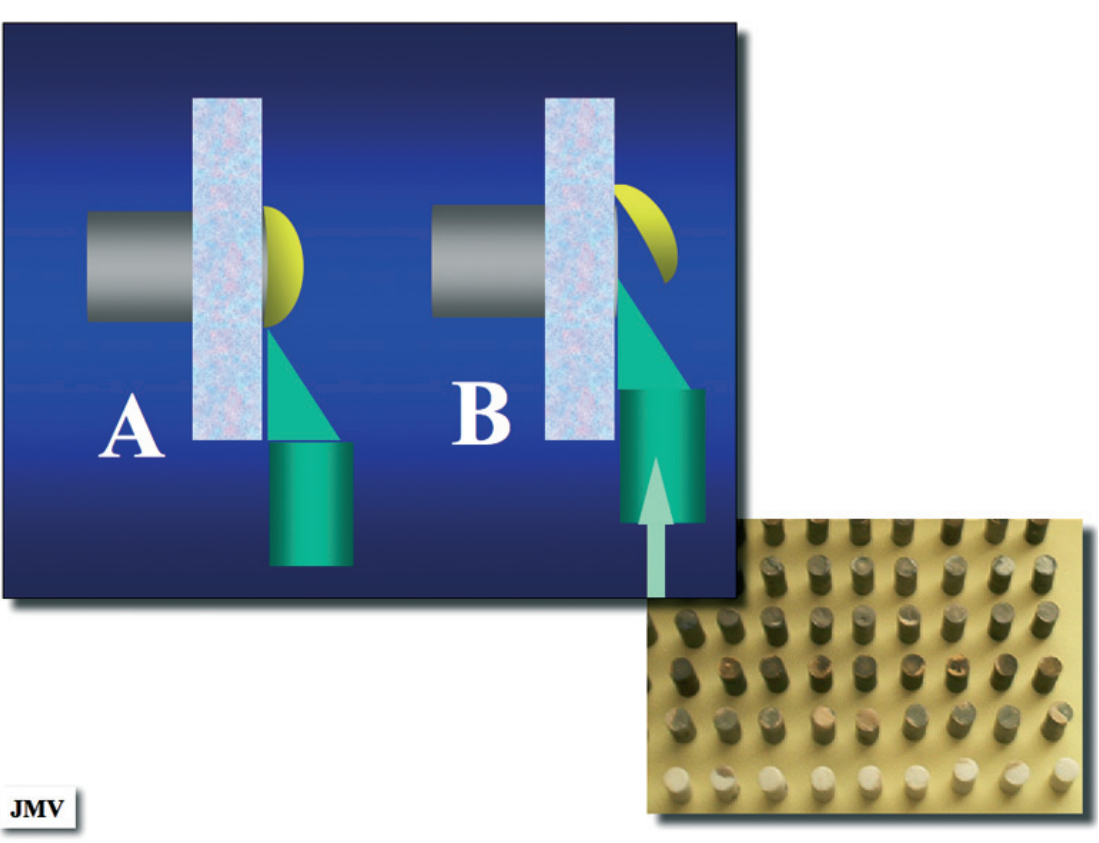

Figura 8. Arriba a la izquierda, representación esquemática de la prueba de cizallamiento. Abajo, a la derecha, algunas de las probetas después de sometidas a la carga de cizallamiento.

\begin{tabular}{|c|c|c|c|c|c|c|c|c|}
\hline \multicolumn{2}{|c|}{ Probeta } & \multicolumn{7}{|c|}{ Valores obtenidos en cada grupo (Mpa) } \\
\hline $\mathrm{n}^{\circ}$ & SN & 1 & II & III & IV & V & VI & VII \\
\hline 1 & 42,71 & 21,84 & 36,28 & 33,35 & 26,33 & 33,55 & 71,78 & 43,44 \\
\hline 2 & 45,64 & 36,86 & 32,38 & 20,09 & 32,01 & 43,88 & 50,13 & 26,59 \\
\hline 3 & 46,03 & 27,89 & 38,42 & 38,03 & 31,42 & 41,93 & 63,78 & 44,06 \\
\hline 4 & 34,13 & 30,82 & 34,72 & 60,85 & 22,64 & 47,01 & 38,03 & 46,74 \\
\hline 5 & 34,52 & 24,57 & 18,53 & 31,6 & 45,67 & 64,76 & 32,96 & 45,56 \\
\hline 6 & 32,18 & 8,97 & 20,48 & 47,2 & 48,6 & 14,04 & 43,88 & 49,81 \\
\hline 7 & 37,64 & 23,4 & 38,23 & 32,38 & 33,18 & 34,33 & 45,05 & 33,83 \\
\hline 8 & 34,52 & 32,02 & 30,42 & 40,18 & 23,03 & 32,77 & 49,54 & 33,58 \\
\hline 9 & 55,59 & 25,16 & 22,82 & 37,25 & 25,57 & 49,93 & 49,54 & 56,17 \\
\hline 10 & 38,62 & 30,23 & 19,5 & 33,94 & 18,92 & 40,96 & 33,55 & 38,45 \\
\hline Media & 40,16 & 26,18 & 29,16 & 37,49 & 30,74 & 40,32 & 47,82 & 41,82 \\
\hline s.d. & 7,31 & 7,57 & 8,05 & 10,75 & 9,78 & 13,25 & 12,40 & 8,76 \\
\hline
\end{tabular}

a la máquina universal para ensayos mecánicos y permanece fija durante el experimento. La parte inferior de la máquina, que es la que produce el movimiento de aproximación, va aco- plada a una platina de sección prismática triangular, de forma que una de las aristas es la que incide sobre la faceta de porcelana, como queda representado en el esquema de la figura 8.
- Equipamiento informático constituido por un ordenador IBM acoplado a la máquina de ensayos mecánicos y el software específico para obtener los datos mediante pantalla e impresora.

Los especimenes confeccionados se llevaron a la máquina universal para ensayos mecánicos. Una vez instalados los aditamentos necesarios se procedió al ensayo específico de cizallamiento, esquematizado en la parte superior izquierda de la figura 8. Todos los cálculos están hechos para expresar los resultados en megapascales. Las condiciones del experimento fueron temperatura ambiente $22^{\circ}$ C; humedad relativa $35 \%$; velocidad del test: $0,1 \mathrm{~mm} / \mathrm{min}$.

\section{Resultados}

Durante el procedimiento experimental, se siguió una técnica doble ciego de tal forma que el laboratorio preparador de las muestras y el laboratorio experimental se comunicaban mediante un código con números romanos, números árabes y/o letras (ver tabla 2). La identificación quedaba guardada en un sobre. En la tabla 2 se recogen los datos obtenidos para cada uno de los grupos experimentales de 10 muestras cada uno.

Una vez finalizadas todas las mediciones se procedió a abrir los sobres identificativos. En la tabla 1 se refleja un resumen de dicha identificación: Grupo SN: se trata de una aleación de níquel-cromo a cerámica clásica (Ticonium + Ceramco II). Grupo I: se trata de un sistema de titanio a cerámica mediante una combinación de materiales experimental, de prueba, cons- 


\section{Resistencia al cizallamiento: medias de 8 sistemas medidos en MPa}

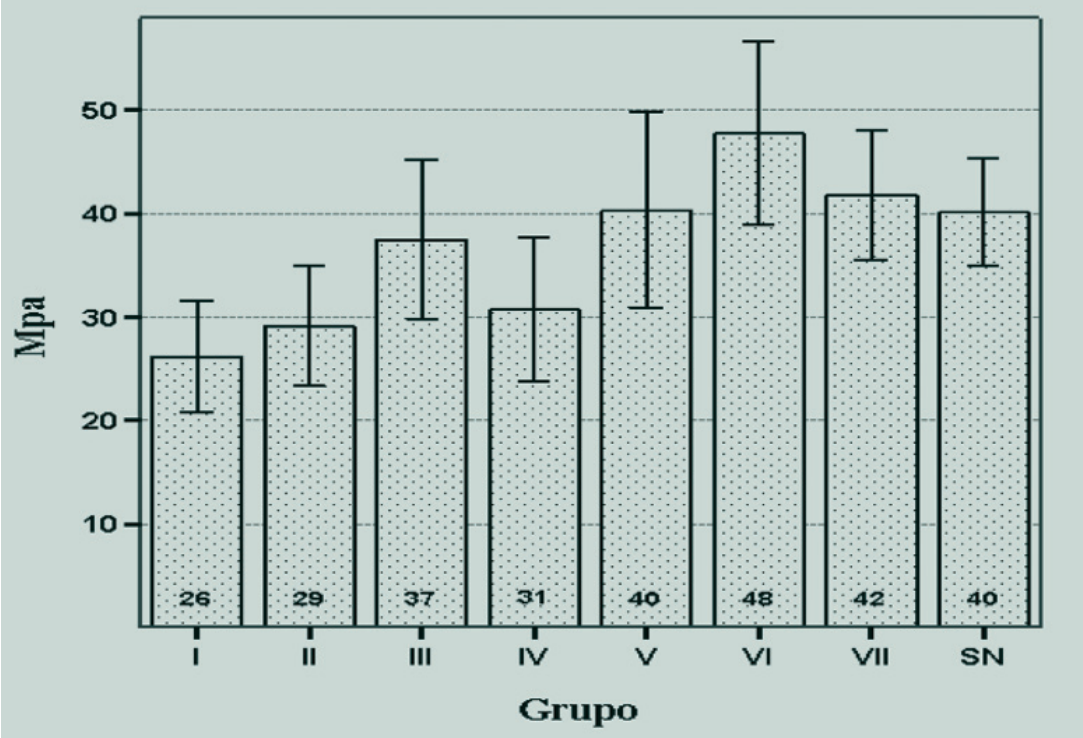

Figura 9. Representación gráfica de las medias de los 8 sistemas probados. El grupo VI es el que consigue los valores absolutos mas altos: es el totalmente cerámico (Cercón).

tituido por Titanio + Triceram (Dentaurum) y bonding (Heraeus). Grupo II: se trata de un sistema específico de titanio a cerámica Vitatitan + Vitakeramik de Vita y bonding específico del sistema. Grupo III: se trata de un sistema específico de titanio colado unido a cerámica constituido por Rematitán + Triceram (Dentaurum) y bonding específico del sistema. Grupo IV: se trata de otra combinación de prueba a base de titanio colado + cerámica Noritake y bonding de Heraeus. Grupo V: se trata de una aleación más moderna de níquel-cromo (con una pequeña cantidad de titanio) a cerámica (Tilite + Ceramco II). Grupo VI: se trata de un sistema ceramo-cerámico o totalmente cerámico Cercon base + Cercon Ceram S (Cercon Smart Ceramics). Grupo VII: se trata de una aleación de cobalto-cromo a cerámica (Sheradent + Ceramco II).

El análisis estadístico se ha realizado mediante el programa SPSS 13 de la SPSS ${ }^{\circledR}$ Inc. Chicago Illinois, U.S.A. EI test de ANOVA indicó que hay diferencias estadísticamente significativas entre las medias de los grupos ( $p=$ 0,00005).

En la figura 9 se refleja la estadística descriptiva. La tabla 3 muestra los resultados del test de Student NewmanKeuls para $p=0,05$. Hay diferencias estadísticamente significativas entre el grupo experimental estudiado (VI) y los grupos I, II y IV.

Como puede apreciarse, la media mas alta, en valores absolutos, corresponde al grupo VI (totalmente cerámico) con un valor medio de 47,82 Mpa. Desde el punto de vista de lo aquí representado constituye la mejor alternativa, en cuanto a resistencia mecánica, frente a todas las uniones ceramometálicas estudiadas (tabla 2 y fig. 9).

Hay tres sistemas cuyos valores siguen en importancia al sistema totalmente cerámico. Se trata de las dos uniones $\mathrm{Ni}-\mathrm{Cr}+$ cerámica (sistema SN y sistema V) y la unión Co-Cr + cerámica (sistema VIII). Son, además, los que ofrecen los mejores resultados de entre los ceramo-metálicos. Los valores absolutos de ambos sistemas $\mathrm{Cr}-\mathrm{Ni}+$ cerámica son prácticamente idénticos.

Esto plantea varios temas de interés. Por una parte se confirma lo que es sabido desde hace muchos años: mecánicamente, las opciones níquelcromo + cerámica, entre las ceramometálicas, son las que ofrecen mejores perspectivas. Por otra parte, y dado que el níquel es objeto de diferentes trabas por sus problemas de posibles alergias, una alternativa totalmente válida, desde el punto de vista mecánico, es la que representa la unión cobalto-cromo+cerámica (grupo VII), independientemente de lo ya comentado para el sistema totalmente cerámico (grupo VI)

Por lo que respecta a los cuatro sistemas de unión del titanio a la cerámica, son los que han obtenido los valores más bajos (I, II, III y IV). El que comparativamente sean más bajos no significa que clínicamente sean desechables. Se trata de un estudio que solamente mide y compara valores, pero de ello no puede deducirse que las uniones ceramometálicas entre titanio y cerámica sean ineficaces a la hora de resistir las cargas masticatorias. 
Otro aspecto a considerar es el de la dispersión de los valores máximos y mínimos encontrada dentro de algunos grupos (tabla 2 y fig. 9). Esto podría indicar que estos sistemas son sensibles a la técnica de manejo.

Una última consideración: ¿Fractura cohesiva o fractura adhesiva? En la simple inspección visual de las muestras (fig. 8), una vez sometidas a las pruebas de cizallamiento, se aprecian diferencias entre unos especimenes y otros, dentro cada grupo y entre las diferentes muestras. Solamente se desea dejar constancia de ello. Se estima que puede ser un material interesante objeto de un estudio independiente aparte.

\section{Discusión}

Esta investigación persigue comparar la resistencia al cizallamiento de una unión ceramo-cerámica, de reciente incorporación al mercado (sistema Cercon Smart Ceramics $®$ ), frente a siete diferentes uniones ceramo-metálicas. El conjunto está constituido por un total de 8 grupos. Cada grupo consta de diez especimenes, lo que globaliza 80 muestras. Como se indicó al principio se ha medido la resistencia a cargas de cizallamiento de la unión entre:

A) Dos sistemas níquel-cromo a cerámica (GRUPO SN y GRUPO V).

B) Cuatro sistemas titanio a cerámica (GRUPO I, GRUPO II, GRUPO III y GRUPO IV)

C) Un sistema totalmente cerámico o ceramo-cerámico (sistema CERCóN SMART CERAMICS $\left.{ }^{\circledR}\right)$ (GRUPO VI) ).

D) Un sistema cobalto-cromo a cerámica (GRUPO VII).

\begin{tabular}{|c|c|c|c|c|}
\hline & & $\begin{array}{l}\text { bla. } 3 . \\
\text { لl New }\end{array}$ & $\begin{array}{c}\text { ast de } \\
\text { dn-He }\end{array}$ & \\
\hline & & conjun & para a & $=.05$ \\
\hline Grupo & $\mathrm{N}$ & 1 & 2 & 3 \\
\hline 1 & 10 & 26,1 & & \\
\hline$\|$ & 10 & 29,1 & 29,1 & \\
\hline IV & 10 & 30,7 & 30,7 & \\
\hline III & 10 & 37,4 & 37,4 & 37,4 \\
\hline SN & 10 & & 40,1 & 40,1 \\
\hline V & 10 & & 40,3 & 40,3 \\
\hline VII & 10 & & 41,8 & 41,8 \\
\hline $\mathrm{VI}$ & 10 & & & 47,8 \\
\hline Sig. & & 0,062 & 0,062 & 0,150 \\
\hline
\end{tabular}

Esa discusión puede plantearse desde dos puntos de vista. Uno se centra en el método escogido para aplicar las fuerzas; otro, en los diferentes materiales empleados para ser comparados. En los trabajos revisados centramos la atención en estos dos aspectos ya que algunos de ellos investigan también otros muchos temas tales como morfología de la superficie del corte mediante microscopía de barrido, resistencia en función del calor del molde durante el colado metálico, otros procedimientos de aplicar las cargas, etc., o se refieren a comparaciones entre técnicas ceramometálicas o a técnicas con resinas compuestas.

La resistencia de los materiales, en general, es estudiada de muy diferentes maneras. Hay estudios con cargas o fuerzas de compresión (axiales o anguladas), de flexión en tres puntos o cuatro puntos, de deslizamiento, cizallamiento, tracción y torsión. Para el presente estudio hemos escogido la prueba de cizallamiento por considerar que reproduce muy bien muchas situaciones de la dinámica máxilo-mandibular sobre muchas restauraciones. Entendemos por cizallamiento aquella situación en que el material (o materiales) son sometidos a la acción de dos fuerzas opuestas que se aproximan entre sí sobre diferentes rectas paralelas ${ }^{6^{*}}$. Con tal fin se diseñaron específicamente para este experimento los dispositivos mostrados en las figuras 7 y 8 . En la revisión bibliográfica realizada hemos encontrado diversos trabajos que investigan la resistencia de la unión entre pares de materiales (metal-cerámicos, totalmente cerámicos o de resinas compuestas a metales) pero no hemos encontrado ninguno en que se realice la comparación propuesta por nosotros entre un sistema totalmente cerámico y siete metal-cerámicos. Aunque en todos los trabajos consultados se mide la resistencia de la unión entre diferentes materiales hay variaciones relevantes en cuanto a la disposición y elaboración de los especimenes y a su tamaño. Nosotros hemos escogido una superficie de contacto, entre los materiales a estudiar, de $8 \mathrm{~mm}$ de diámetro (alrededor de 50 milímetros cuadrados) por considerar que se trata de una superficie compatible con el uso clínico de los materiales en una cara dental libre. Los ensayos puros de compresión o de carga en tres puntos (flexión), que aparecen con frecuencia en la literatura consultada, van encaminados a simular in vitro las condiciones bucales de los puentes pero no estudian el comportamiento de las fuerzas de deslizamiento, tangenciales y de cizallamiento que aparecen en diferentes zonas laterales de las restauraciones, sobre todo en las interfases entre materiales. 
En el trabajo de Halmihatti y cols utilizaron una metodología muy parecida a la descrita en el presente trabajo. El diámetro de la superficie de unión entre materiales fue también de $8 \mathrm{~mm}$. La aleación empleada fue una de Ni-Cr pero la comparación la efectúan frente a materiales unidos al metal que son resinas, ormoceras y cerómeros (Artglass, Solidex y Targis) frente a porcelana unida a metal (Noritake + NI-Cr). En una de sus conclusiones se menciona que la porcelana unida a metal muestra una resistencia de la unión al deslizamiento considerablemente más alta que los tres sistemas de resina. Ku y cols ${ }^{8}$ también compararon la resistencia de grupos de coronas ceramometálicas frente a coronas realizadas con cerómeros (Artglass, sculpture y Targis) que recibieron cargas con una determinada angulación. En sus resultados encontraron también mayor resistencia de las coronas ceramometálicas.

En un trabajo de Al-Dohan y cols ${ }^{9}$ se utilizaron cilindros en los que la superficie de unión entre los pares de materiales era de 2,4 mm. Investigaron la resistencia de la unión al cizallamiento de tres sistemas totalmente cerámicos frente a uno ceramo-metálico. Entre los sistemas cerámicos empleados incluyeron dos con zirconia (el otro es IPS-Empress 2+Eiris). Los resultados muestran que no hay diferencias significativas entre los sistemas totalmente cerámicos y el metal-cerámico.

Bondioli y cols $^{10}$ utilizaron fuerzas de deslizamiento o cizallamiento pero con un diseño experimental diferente al nuestro. La prueba de resistencia de la unión se efectuó sobre el material cerámico recubriendo la superficie lateral, cilíndrica, de las estructuras metálicas. Aunque utilizaron dos sistemas de cerámica unida al titanio similares a los nuestros (Triceram y Titankeramic), se centraron mas en investigar las variaciones que pueden introducir en la resistencia de la unión entre titanio puro y cerámica, las diferencias de temperatura del molde durante el colado del metal.

Hay un estudio extenso e interesante de Guazzato y cols ${ }^{11,12}$, publicado en dos partes, que recoge una amplia experimentación sobre resistencia a la fractura y microestructura de una selección de materiales totalmente cerámicos entre los que se encuentran algunos constituidos por zirconia. Se trata de ensayos constituidos por carga en tres puntos.

Otros autores consultados investigaron sobre resistencia de la unión entre metal y cerámicas utilizando metodologías ya mas alejadas de la nuestra o la resistencia de la unión entre metales y materiales a base de resinas compuestas. Así, Filser y cols ${ }^{13}$ estudiaron la resistencia de estos materiales sobre un modelo experimental de puentes de tres piezas aplicando cargas de flexión. Esta situación experimental es distinta a la nuestra y aunque se comparan dos sistemas totalmente cerámicos, ninguno de ellos fue contemplado en nuestro ensayo (In-Ceram Alúmina e IPS-Empress 2). Ludwig ${ }^{14}$ hace alusión a la resistencia a la fractura de coronas de cerámica sin metal pero utilizó grupos de coronas completas a las que aplica cargas de compresión de dirección axial y de dirección oblicua respectivamente. Comparó los sistemas IPS-Empress y Dicor con una cerámica convencional.
En un trabajo, algo mas alejado en el tiempo, Persson y Bergman ${ }^{15}$ realizaron un complejo estudio en el que compararon la resistencia al cizallamiento de sistemas ceramo-metálicos de titanio mecanizado y de titanio colado frente a sistemas ceramo-metálicos en que el metal era una aleación de alto contenido en oro. Incluyeron una comparación de resultados con el estudio de resistencia en una corona ideal de elementos finitos. En sus conclusiones encuentraron que hay mayor resistencia de la unión de los dos sistemas con titanio que con la aleación de alto contenido en oro. Otros estudios, como los de Petridis y cols ${ }^{16}$, o los de Cilbert y cols ${ }^{17}$ se alejan de los objetivos perseguidos en el presente trabajo.

En cuanto a los materiales comparados, la selección que hemos realizado parece muy apropiada al momento presente ya que la revolución que suponen la llegada de nuevos materiales totalmente cerámicos puede marcar un hito importante en el mundo odontológico frente a los sistemas metal-cerámicos para este tipo de restauraciones. Este trabajo de investigación in vitro tiene la ventaja de que puede realizarse con una metodología relativamente sencilla de elaborar y ejecutar.

\section{Conclusiones}

A la vista de los resultados obtenidos en la presente investigación se pueden obtener las siguientes conclusiones:

1. En valores absolutos, los valores medios mas altos, frente a cargas de cizallamiento, se obtienen para el grupo totalmente cerámico (Cercon 
Smart Ceramic) frente a todos los grupos ceramo-metálicos estudiados.

2. No obstante solo aparecen diferencias estadísticamente significativas entre el grupo experimental o de prueba estudiado (VI) (Cercón) y los grupos I, II y IV.

3. Desde el punto de vista mecánico la elección del sistema totalmente cerámico estudiado (Cercón ${ }^{\circledR}$ ) es la alternativa más válida frente a las opciones ceramometálicas I, II y IV.
4.También desde el punto de vista mecánico, en aquellos pacientes que presenten algún problema con el níquel (alergias), son alternativas válidas tanto el sistema totalmente cerámico estudiado como el Co-Cr + cerámica (grupo VII).

5. En alguno de los sistemas estudiados se aprecian oscilaciones de cierta importancia en las desviaciones estándar. Dadas las diferencias dentro de algunos sistemas, ello parece indi- car que hay sistemas muy sensibles a la técnica de manejo.

\section{Agradecimientos}

El autor agradece la colaboración y la profesionalidad de todos los componentes, tanto del equipo directivo como del personal técnico de Laboratorios Aragoneses S. L., en la preparación de las muestras empleadas en el presente ensayo.

\section{Bibliografia recomendada}

Para profundizar en la lectura de este tema, el/los autor/es considera/an interesantes los artículos que aparecen señalados del siguiente modo: *de interés ${ }^{*}$ de especial interés.

1. Anusavice KJ. Cerámicas dentales. En: Anusavice KJ. Phillips Ciencia de los Materiales Dentales. Undécima edición. Madrid: Elsevier, 2004.

2. Salsench J, Cabré J, Gascon FJ y cols. Puentes experimentales totalmente de cerámica. Bases y proceso de confección. Quintessence (ed esp) 1991;4:467-71.

3. Vega JM. Cerámica dental. En Toledano M. y cols.: Arte y Ciencia de los Materiales Odontológicos. Madrid: Ediciones Avances, 2003:28396.

4. Vega JM. Uniones ceramo-metálicas y ceramocerámicas: perspectivas futuras. Gaceta Dental 2004;149:106-14.

5*. Alvarez MJ, Peña JM, Gonzalez I-R, Olay MS Características generales y propiedades de las cerámicas sin metal. RCOE 2003;8:525-46. En las cinco referencias bibliográficas precedentes el lector interesado puede encontrar amplia documentación sobre los desarrollos modernos del complejo mundo de las cerámicas.

6*. Vega JM. Propiedades físicas mecánicas de los materiales odontológicos (I). En Vega JM: Materiales en Odontología. Fundamentos biológicos, clínicos, biofísicos y fisicoquímicos. Madrid: Ediciones Avances, 1996: 61-75. En este capítulo se puede encontrar información general sobre los principios generales relativos a ensayos tecnológicos aplicados a la resistencia de los materiales odontológicos.

7. Almilhatti HJ, Giampaolo ET, Vergani CE Machado AL, Pavarina AC. Shear bond strength of aesthetic materials bonded to $\mathrm{Ni}$ Cr alloy. J Dent 2003;31:205-11.

8. Ku CV, Park SW, Yang HS. Comparison of the fracture strength of metal-ceramic crowns and three ceromer crowns. J Prosthet Dent 2002;88:170-5.

9. Al-Doan HM, Yaman P, Dennison JB, Razzoog ME, Lang BR. Shear strength of core-veneer interface in bi-layered ceramics. $J$ Prosthet Dent 2004;91:349-55.

10*. Bondioli IR, Bottino M. Evaluation of shear bond strength at the interface of two porcelains and pure titanium injected into the casting mold at three different temperatures. J Prosthet Dent 2004;91:541-7.

Los cuatro artículos precedentes ofrecen información específica sobre aspectos directamente ligados al contenido del presente trabajo.

11. Guazzato M, Albakry M, Ringer SP, Swain MV Strength, fracture toughness and microstructure of a selection of all-ceramic materials. Part I . Pressable and alumina glass-infiltrated ceramics. Dent Mater 2004;20:441-8.

12. Guazzato M, Albakry M, Ringer SP, Swain MV Strength, fracture toughness and micros- tructure of a selection of all-ceramic materials. Part I I. Zirconia-based dental ceramics. Dent Mater 2004;20:449-56.

13. Filser F, Kocher P, Weibel F, Lüthy H, Schärer $P$, Gauckler LJ. Reliability and strength of AllCeramic Dental Restorations Fabricated by Direct Ceramic Machining (DCM). Int J Comput Dent 2001:4:89-106.

14. Ludwig K. Análisis de la resistencia a la rotura de las coronas de cerámica sin metal (traducción al español). Dental-labor 1991;5:64751.

15. Persson M, Bergman M. Metal-ceramic bond strength. Acta Odontol Scand 1996;54: 160-5.

16. Petridis H, Garefis P, Hirayama H, Kafantaris NM, Koidis PT. Bonding indirect resin composites to metal: Part 1. Comparison of shear bond strengths between different metalbonding systems and a metal-ceramic system. Int J Prosthodont 2003; 16:635-9.

17*. Gilbert JL, Covey DA, Lautenschlager EP. Bond characteristics of porcelain fused to milled-titanium. Dent Mater 1994;10:134-40. En estas últimas siete citas bibliográficas se pueden encontrar diferentes trabajos experimentales que investigan, desde diferentes ópticas, la resistencia de la unión metal-cerámica, ceramo-cerámica o de resinas compuestas, ormoceras, cerómeros, etc. 